\title{
On “New Retailing” Strategy of New Hua Du Traditional Supermarket
}

\author{
Herong Zhang \\ School of Economics and Management \\ Minjiang University \\ Fuzhou, Fujian, 350108
}

\begin{abstract}
The research is carried on the traditional supermarket that adopts "new retailing" business model. This paper analyzes the difficulties of the traditional supermarket and the necessity to keep running under the "new retailing" strategy. The "new retailing" strategy of the New Hua Du supermarket was studied in terms of the technical, organizational, and time feasibility, and corresponding development approaches and suggestions are put forwarded to promote the new business mode and enhance the overall competitiveness of traditional supermarkets.
\end{abstract}

Keywords—new retailing; traditional market; chain selling; consumer experience

\section{INTRODUCTION}

First mentioned in October, the "new retailing" was released in the Computing Conference together with other concepts like "new finance, new manufacturing, new technology, and new energy". As for the new retailing, Jack Ma has given the interpretation that it is a data-driven reconfiguration of the three elements of business: people, goods and market. [1] That is to say, it needs to rely on internet technology, integrate the online business, offline dealings and logistics to improve consumers' experience. The reconfiguration of people, goods and market will generate a brand-new retailing mode which will be the orientation of traditional retailers.

Recent years have witnessed the slump of traditional supermarket against the rapid development of e-commerce. As shown in Fig. 1, the average profit increase rate is on the decline in these years. Some popular supermarkets even showed negative growth in net profit in 2016. The whole retail industry was in a sluggish state.

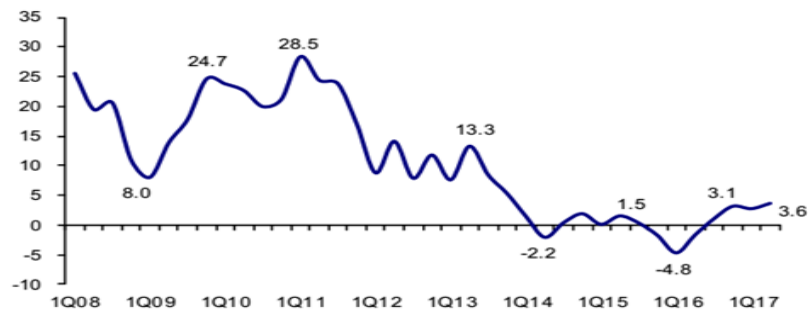

Fig. 1. Quarterly Growth in Retail Sales of 100 Large Enterprises (\%, 1Q2008-2Q2017)

Fund Projects:

Subject Project of Mingjiang University in 2018 (No. MJW201833215),

Subject Project of Mingjiang University in 2018 (No. XTXZ1812),

Subject Project of Mingjiang University in 2019 (No. MYVS2019B003)
Under this circumstance, some large-scale supermarkets that used to dominate varied markets are shut down, such as Jiahe and Baiquan. Facing with new challenges, they are risk of bankruptcy if they do not seek new possibilities. In fact, there are some retail entities or enterprise coming to turn to ecommerce, and cooperation among industries in order to survive and keep developing.

At present, China's overall economic growth slows down, the national retail enterprise revenue growth rate is declining. Moreover, with the emergence of a prosperous e-commerce platform, the development space of traditional retail commerce is being swallowed up step by step. However, it is undeniable that the traditional retail industry still has its own advantages, which is difficult for e-commerce to surpass, and online retail cannot completely replace the traditional retail business. As shown in Fig. 2, although the online retail sales are increasing year by year, the growth rate is slowing down. While enjoying their own growth, online retail enterprises should also pay attention to the slowdown of growth rate and seek for a better development mode. Both traditional and online retail businesses have their own limitations. Only the new retail business model with online and offline cooperation can bring more profits to enterprises. The integration of both and their functioning differently are inevitable for satisfying individuation and diversification in consumer consumption demand.

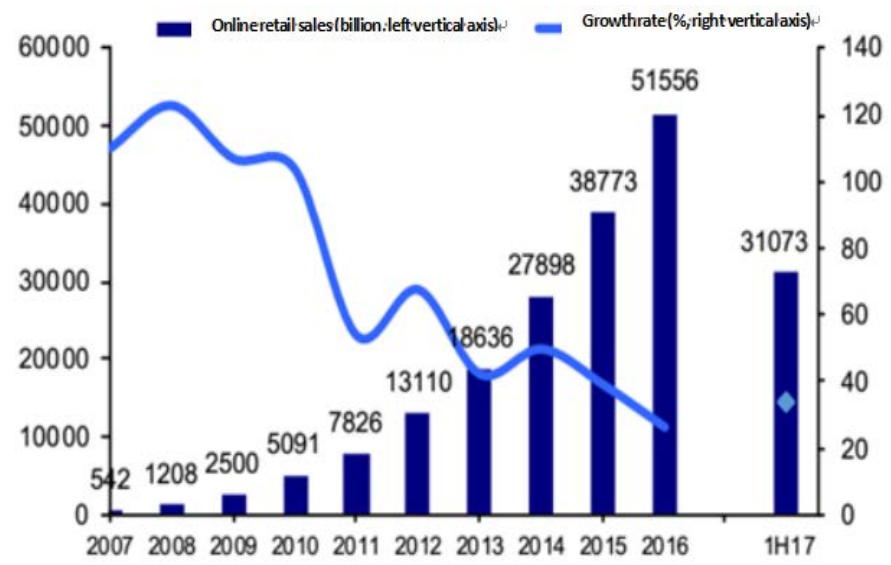

Fig. 2. Growth of Online Retail Sales (2007-1H2017) 
II. THE DEVELOPMENT OF TRADITIONAL SUPERMARKETS AND THE NECESSITY OF DEVELOPING “NEW RETAIL” STRATEGY

\section{A. Analysis of the advantages of traditional supermarkets}

In the information era where traditional supermarkets are uncompetitive, the supermarkets that still occupy the independent part of the market are attributed to their own advantages.

\section{1) Advantages of external environment}

(1) The level of consumption in China is constantly improving, so people are spending on consumer goods. That is undoubtedly a development opportunity for traditional supermarkets.

(2) In recent years, the Chinese government has done more to help develop rural areas, increasing the consumption in rural areas by improving the infrastructure facilities. That can be a potential market for supermarkets to explore.

The central bank has lowered the loan interest rate and the deposit reserve to support the healthy and sustainable development of the real economy. At the same time, the state supports the emerging industries by issuing many regulations on financing and taxation of e-commerce development, which provides policy support and guarantee for the development of "new retail" in traditional supermarkets.

2) Advantages of the internal environment

(1) Shopping environment. Traditional supermarkets do a good job in display of goods, the configuration of facilities, service attitude and other aspects, giving consumers better shopping experience.

(2) Local culture. Traditional supermarkets, such as New Hua Du and Yonghui, have a good understanding of local residents' consumption habits, consumer psychology and other information, and all have their own fresh product bases in the local area, which can best meet the needs of customers.[3]

(3) Brand effect. The traditional supermarkets promote their own reputation in the operation, gaining a number of loyal customers that trust their products.

(4) The collections of products satisfy consumer psychology. Many customers may buy goods that are not expected when shopping, which virtually increases the turnover of the supermarket.

(5) Mature management. The supermarket has experienced a long period of precipitation since its formation and has a mature management mode.

\section{B. Problems faced by traditional supermarkets}

Today, with the deepening of information technology in every dimension of society, people's demand and purchasing are changing. Although the traditional supermarket occupies an important part of the market, they also face some problems that cannot be ignored.
1) The influence of social environment on traditional supermarkets

(1) Since 2012, the growth rate of China's GDP has gradually fallen, no longer the rapid development lasting for more than 30 years. The essence of economic development in this period is to make people get happiness, optimize the economic structure. In such a macroeconomic environment, the development of traditional supermarkets will be relatively difficult.

(2) The way people spend money has changed. At present, the main consumers are the post-80s and post-90s young people who prefer online shopping and one-stop service with leisure, shopping and entertainment. This is a huge shock to traditional supermarkets.

(3) In the information age, emerging industries and new shopping modes come into being. Traditional supermarkets should seek their own development path, or they will be easily eliminated.

(4) The implementation of anti-corruption initiative brings down sharply the sales of pick-up and gift cards in supermarkets, as well as sales of some luxury goods. Traditional supermarkets should seek a long-term, sound operation mode.

2) Market competitive position is being choked

Traditional supermarkets are threatened by both the foreign retail enterprises who want to take a share of the Chinese market, and the domestic online retailers who leverage price advantage to seize the market.

\section{3) Traditional supermarket management problems}

Most supermarkets of different enterprises are located in the busy areas of the city. Moreover, the homogenization of goods results in no other marketing mode but the price competition. There are also some problems in the management of traditional supermarkets, such as poor leadership, poor staff quality and so on.

\section{The necessity of implementing "new retail" strategy}

Traditional retailers have been suffering and trying to make a breakthrough in order not to be squeezed out of the market these days. Although many ways have been tried, such as "Internet +", online and offline O2O, etc. the retailers did not get out of trouble due to the weak strength, in adaptation or bad learning. Therefore, it is an irresistible trend to actively look into the current environment and develop the strategy of "new retailing".

1) The support of national policies

In November 2016, the state council issued Opinions on Promoting Innovation on and Transformation of Physical Retail which proposes to encourage online and offline enterprises to integrate market resources through strategic cooperation, cross-shareholding, merger and reorganization and other forms, and cultivate new market subjects for integrated online and offline development. The enterprises must pay attention to national policies and regulations to make development strategy and make innovations. The strategy of 
"new retailing" complies with the national policy and suits the pursuit and development of supermarkets.

\section{2) The support of modern technologies}

The constant development of modern technology, the arrival of the era of big data, the development of advanced technologies such as artificial intelligence and modern logistics, all provide technical support for the development of "new retailing” strategy.

\section{3) Characteristics of the retail industry}

First, most consumers are price-sensitive, so they may turn to e-commerce platforms where there are plenty of affordable products. Second, most traditional supermarkets sell necessities of life, and there are many substitutes for these commodities. So, they are threatened by substitutes. Third, due to the development of information technology and the low threshold of the retail industry, those who have more advanced technologies can provide better purchasing experience for consumers. In a word, as competition in the retail industry gets fierce, traditional supermarkets need to find ways to survive.[2]

4) The development needs of traditional retail enterprises

First, the backward management mode of traditional retail enterprises no longer suits modern enterprises, so traditional retailers must keep pace with the Times. Second, in the Internet era, enterprises' increasing demand for information and data puts traditional retail enterprises at a disadvantage.

\section{5) Consumer analysis}

In recent years, with the improvement of consumption level and the transformation of consumption form, consumers pay more attention to product quality and service. Online and offline retailers are striving to improve consumers' experience, but they cannot make substantial improvement on themselves. However, "new retailing” can provide consumers with superior consumer experience and meet their needs.

\section{NEW HUA DU COMPANY “NEW RETAILING” STRATEGY ANALYSIS}

\section{A. New Hua Du company operating status}

The huge storm in the traditional retail market in the past few years has also affected New Hua Du. In the past two years; it has adopted a series of measures to improve itself. The following are the analysis of the main business income of New Hua Du and the analysis of financial statements and other data that show its current operating status.

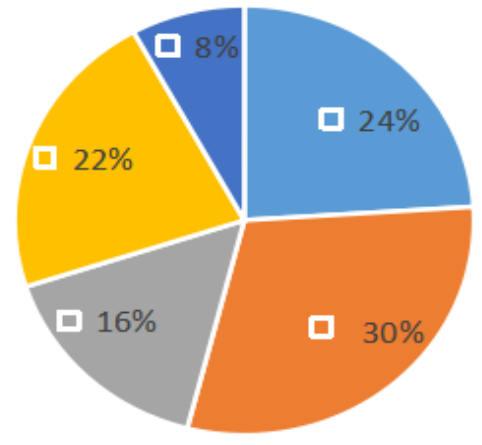

" Fresh products $\mid$ Food $\mid$ Commodity $\mid$ Non-food $\mid$ Others

Fig. 3. New Hua Du Main Businesses Proportion

As shown in Fig.3, the operation mode of New Hua Du is "hypermarket + department store". The income is mainly from department stores and daily necessities, accounting for about $45 \%$ of the total revenue. It just differs from Yonghui supermarket which runs with fresh products, avoiding the direct competition of the local market in Fuzhou.

TABLE I. 2016-2018 NEW Hu Du PERFORMANCE REPORT

\begin{tabular}{rrrrrrrr}
\hline Date & Operation Revenue & Operation Profit & Total Profit & Total Assets & ROEWA & net asset value per share \\
\hline 2016 & 6709549178.27 & 18752913.91 & 39176398.88 & 3742359871.69 & $3.37 \%$ & 2.48 \\
2017 & 6972538773.56 & 8599879.35 & -17490558.16 & 3518597625.04 & $-3.14 \%$ & 2.40 \\
2018 & 6850013703.42 & 50248782.68 & 50924788.87 & 3631456452.94 & $3.21 \%$ & 2.42 & \\
\hline
\end{tabular}

The operating income of New Hua Du (as shown in table I) in recent three years is affected by the general environment. In 2016, the impairment of assets of New Hua Du was mainly attributed to real estate projects, but the original retail industry turned losses into gains, and the overall trend was developing in a good direction.[3] In 2017, the growth of operating income was mainly due to the growth of main business performance, but the return on equity was reduced due to investment and business losses. In 2018, it made more efforts in the optimization and integration of supply chain, reduced costs and improved comprehensive gross profit rate. As can be seen from the table, New Hua Du attempting to pursue long-term development should keep pace with the Times, and constantly seek change and innovation.

\section{B. New Hua Du “new retailing” strategy}

\section{1) Actively develop agent business}

On June 16th, 2015, New Hua Du acquired Jiuaizhihe, Jiuaitianjin and luzhouzhihe, realizing the integrated development of traditional retail and e-commerce by taking advantage of the Internet thinking and big data of e-commerce companies. Since then, New Hua Du has vigorously developed the E-commerce agent operation which is mainly on the liquor industry. It promotes offline retail through the "passive" sinking of online popular products and takes the initiative to expand the rural market with Alibaba and JD, applies the "rural agent" similar to weChat business mode, to bind big brand owners. Take the differentiated market positioning, and use the production capacity of these brand owners to develop and 
formulate new products together. At present, certain progress has been made.

\section{2) To strengthen refined enterprise management}

New Hua Du also carries out refined management, including "Linju” life supermarket, maternal and infants store, Jiajiaduo, Dudu Snacks and so on. "Linju" community supermarket meets the personalized, fragmented, convenient consumption needs. Its "Yinte Sports" actively carries out parties and sports events among young generations, and introduces young teams to increase brand vitality and customer viscosity, making it more suitable for current consumer demand.

\section{3) To expand new business models}

In the new retailing era, Xin Hua Du also integrates the new retailing offline. On May 10, 2017, they made an innovation to introduce the new business pattern of "participation + supermarket", to provide rich varieties, fresh seafood as well as a set of Fujian, Sichuan, and Hunan cuisine, and all kinds of Cantonese refreshments. Diversified restaurant scenes with good environmental quality bring consumer comfortable experience.

\section{4) To strengthen cooperation with large companies}

On September 26th, 2017, New Hua Du announced to sign with Alibaba Chengdu branch, Hangzhou Hanyun on "Stock transfer Protocol", agreeing to transfer $10 \%$ of its shares to them. Moreover, and signed in Alibaba Zetai Hangzhou "The Shareholder Agreement on the Establishment of Fujian Xinhe Network Technology CO., LTD”. The two sides each contributed 100 million Yuan, and each holds 50\% of the stake.

New Hua Du rolls out the "new retailing” strategy through internal reform and external cooperation and independent innovation. By acquiring the e-commerce company, it combines the advantages of e-commerce company with the company's traditional retail to develop the online sales channels, keeping pace with the Times. Cooperate with Alibaba group to realize resource integration by relying on Alibaba's ecommerce edges. In addition, according to the development trend of the new era, the company launches "Linju" supermarket and "Haiwuhui" suitable for the development of the company.

\section{Feasibility analysis of New Hua Du "new retailing”}

1) Technical feasibility analysis

The implementation of new retailing needs the support of big data. In the past two years, through data analysis, Xin Hua $\mathrm{Du}$ found out hot products online to cooperate with other companies. According to the financial statement of 2017, its ecommerce operation performance was good. It can be seen that it is mining and using big data, and by combining big data with its own business operation, drive the development of enterprises.

The integration of online and offline business is indispensable from logistics and distribution. Through the evaluation interfaces of Ele Me and Taobao platform, it can be seen that consumers concern more about quality service, then the logistics. Logistics affects consumers' shopping experience. Therefore, the improvement of logistics distribution mode is also crucial for Xin Hua Du Company which develops the "new retailing" mode. According to the current development status of logistics, JD and Alibaba have a great probability to become the leading logistics and distribution enterprises in the future. Relying on Alibaba's logistics model plays an important role in Xin Hua Du's strategic development.

Now all walks of life in China are developing various technologies to promote the development of mobile internet devices which can bring the convenience of consumption. For example, the popular face recognition technology enables consumers to pay quickly. With the support of Alibaba, the pioneer of the Times, Xin Hua Du may obtain great help in the application of mobile internet device, through the development of which, consumers' stickiness can be improved.

Generally, through cooperation with Alibaba and selfdevelopment, Xin Hua $\mathrm{Du}$ is now capable to apply new technologies to the company's development, which is conducive to improving the company's retail performance.

\section{2) Organizational feasibility analysis}

Through long-term development, Xin Hua Du has a largescale organization and mature management.

Xin Hua Du knows well that the development of an enterprise cannot be achieved without the talents of the new era. In 2010, it donated 500 million yuan to establish New Huadu Business School and later set the Fashu scholarship, aiming to cultivate talents in line with the development of modern enterprises. Through the recruitment information of New Hua $\mathrm{Du}$ this year and the young generation of its leadership, it can be seen that it is introducing a large number of creative young talents to promote the development of its new businesses.

The company's organizational management system is relatively perfect, with clear labor division of departments. It establishes and continuously improves the professional manager system which clearly defines the responsibilities of the board of directors, the general meeting of shareholders and the management staff. The manager culture provides a stable management organization structure for the development of the company.

\section{3) Time feasibility analysis}

The acquisition of three e-commerce companies in 2015 provides big data support for the implementation of "new retailing" strategy in the future. When the online and offline cooperation was booming, New Hua Du turned to Alibaba, an e-commerce giant, to promote the company's development and develop the "new retailing" strategy through in-depth cooperation with Alibaba, seizing the good opportunity of cooperation.

The year 2017 is known as the first year of new retailing, with the emergence of "unmanned supermarket", "retailing + catering" and other new business models. At that time, New Hua Du launched "Haiwuhui" which added the social attributes of traditional retail and improved customers' experience. In the new retailing boom, New Hua Du was rated as China's top 50 new retailers in 2018.

Since Jack Ma proposed the strategy of "new retailing”, the wind of new retailing swept across the country, and each 
representative has his own interpretation of the strategy. For retailers, it is urgent to join the army of "new retailers". The timing is right for New Hua Du to implement its "new retailing” strategy.

\section{SugGESTIONS AND COUNTERMEASURES FOR THE STRATEGIC DEVELOPMENT OF “NEW RETAILING” IN TRADITIONAL SUPERMARKETS}

A. Challenges faced by the traditional supermarkets developing "new retailing" strategy at the present stage

1. Traditional supermarkets are highly homogeneous in terms of commodity categories and supply channels. Due to their lack of differentiation, many supermarkets do not have their own core competitive advantages, and commodity prices become the focus of their competition, which is easy to hurt the vitality of enterprises and cause great pressure and challenges.

2. The "new retailing" strategy is still in the development stage. The strategy is now being practiced for trial. Traditional supermarkets should not be too blind to follow the suit, but to implement strategies in combination with their own conditions, otherwise, it may backfire.

The introduction of "new retailing" is both an opportunity and a challenge for traditional supermarkets. Only by properly examining themselves to find the right path for the development, can the enterprise stand out.

\section{B. Suggestions and countermeasures for the development of traditional supermarkets}

1) To change their management concept to adapt to the changing environment

Traditional supermarket operators should change traditional business philosophy and establish a new business model. Accurately target customers and formulate reasonable and innovative strategic planning. They can learn from Hangzhou Lianhua Huashang Droup's “Jingxuan store” which is a multiscene consumption space where there allows retailing, eating, socializing and entertainment. Always pay attention to the implementation of strategy and landing effect, and find their own distinctive transformation.[4]

2) To become consumer demand-oriented to improve enterprise services

The essence of the "new retailing" strategy is to serve consumers rather than just sell products to them. Traditional supermarkets should pay more attention to consumers' sense of experience and make efforts to develop "smart" stores, to allow customers to experience the convenience of "take and go". The use of modern information technology and data analysis, to get insight into consumer demand, facilitating enterprises to meet the accurate needs of consumers. At the same time, they should control the quality of commodities to provide consumers with high-quality products and services.

3) To improve the technological level of enterprises through cooperation with e-commerce platforms

If transitional supermarkets collaborate with e-commerce platforms, they can realize the following aspect. First, by building online platform or cooperating with online platform, they can optimize all aspects of enterprise operation, expand channel sales, so that consumers can choose products without going out of homes and that what they see is what they get, increasing consumption. Second, the integration of the procurement, inventory and sales, promote the efficiency of the supply chain, which can not only reduce operating costs, but also improve the effectiveness of goods delivery. It is necessary to establish their own logistics system, make use of smart logistics, improve the efficiency and visualization of logistics, and enhance the adaptability and flexibility of the supply of goods, services and formats to the changes of customer demand.[5]

4) Maintain original advantages and build the core competitiveness

Traditional supermarkets have their own characteristics, and many enterprises do not concern about their core competitiveness, thus is easy to be swept away by the tide of The Times. Traditional supermarkets should do a good job in enterprise positioning, recognize the advantages and disadvantages of enterprises and the development orientation, and create unique enterprise core competitiveness. For example, JD logistics is praised and recognized by the public. Alibaba is known for Taobao and Alipay, and Yonghui is fresh products. Only by building core competitiveness, enterprises cannot easily be eliminated in the competition.[6]

\section{CONCLUSION}

With the development of the modernization and information technology, the consumer market keeps upgrading, and consumer demand has been highlighted, showing the characteristics of the community, level, convenience, and service, personalized. In order to better meet the needs of consumers, the retail industry needs constant improvements.

Although online retailing is growing, it has also hit bottlenecks. Meanwhile, offline retailing is difficult to be replaced due to its unique characteristics. However, Alibaba and other online giants have good facilities to develop "new retailing”, such as cloud computing and efficient logistics, which can help traditional supermarkets realize digital operation. It is an inevitable trend for traditional supermarkets to join the army of "new retailing". However, while "new retailing" bring development opportunities to traditional supermarkets, enterprises cannot ignore whether this strategy can be perfectly integrated with enterprises to promote the development of traditional supermarkets. Traditional supermarkets should implement the "new retailing" strategy according to their own characteristics so as to achieve the best strategic effect.

\section{REFERENCES}

[1] Li Zichen. Alibaba's New Retail Resonating around the World [N].International Business Daily, 2017-11-22(8)

[2] Yu Boming, Li Ning. Retailing[M]. Dalian: Dongbei University of Finance \& Economics Press, 2008.

[3] CNII. http://www.chyxx.com/ 
[4] Han Caizhen, Wang Baoyi.The Theoretical Situation of "New Retail" and Its Extension[J]. China Business and Market.2018(12)20-30.

[5] Wu Chaoyun. On the Implementation of Suning's “New Retail” Mode [J]. Journal of Fujian Business University, 2019(2)64-69.
[6] Zhang Yingwen. On Traditional Retail Pricing Strategy Based on "New Retailing" Model [J]. Shandong Textile Economy, 2017(10):46-48. 